\title{
Revisiting the Major Mental Disorders and Updating the Nosological Schema: A Synthesis
}

\author{
Nicholas Pediaditakis \\ Deptartment of Psychiatric Medicine, ECU Brody School of Medicine, Greenville, NC, USA \\ Email: nicholaspediaditakis@gmail.com
}

Received 23 December 2015; accepted 18 February 2016; published 24 February 2016

Copyright ( 2016 by author and Scientific Research Publishing Inc.

This work is licensed under the Creative Commons Attribution International License (CC BY). http://creativecommons.org/licenses/by/4.0/

(c) (i) Open Access

\section{Abstract}

Recently, conciliating findings from molecular genetics, evolutionary biology, along with empirical clinical evidence regarding the major mental disorders (MMDs) namely bipolar affective disorder (BPAD), schizophrenia, obsessive compulsive disorder (OCD), the anxieties with depression, autism and attention deficit/hyperactivity disorder (ADHD) all point to a common neural-developmental origin. Genetic loci associated with schizophrenia do not directly lead to the disorder; instead, they code for the expression of lopsided, temperamental, variants in individuals that originate mainly from one part of our human nature which applies also, to the rest of the MMDs. These individuals contribute to the flexibility, robustness, and creative input of our species, concomitantly, they incur vulnerability to the development of a MMD as an evolutionary trade off. MMDs initially, are expressed as periodic epiphenomena on the underlying temperamental extreme variants of brain function. Their expressions tend to become permanent. Underlying, aberrant traits remain unaltered. Their clinical expressions are characterized by "either-or", antithetical substitutes, in addition to co-occurring psychosis. The latter is a common occurrence to other assaults on brain function. Characteristic, "ether-or" symptoms are the result of a disturbed, overall, coordinating property of brain function, normally responsive to the smooth, synchronizing expression of all higher mental faculties. Clinical findings point to the need of modifying the current schema in order to better reflect their collective significance in order to help guide research to a new, more promising direction in elucidating their triggers, development, and mechanisms whereby opening a new horizon for therapy and treatment.

\section{Keywords}

Major Mental Disorders, Psychopathology, Molecular Genetics, Evolutionary Biology 


\section{Introduction}

Normally, occurring, temperamental variability (i.e., inborn behavioral propensities) confers flexibility and robustness not necessarily to an individual alone, but, to our entire species. As social animals, our evolutionary success depends on it among other factors [1]. It is proposed on the evidence to be addressed that a particular temperamental clustering creates a pool of vulnerable individuals who develop a major mental disorder at a much higher rate to that of general population. This clustering originates mainly, albeit, in its extreme form for this particular group, from evolutionary pressures on the individual. E.O. Wilson and his co-workers discovered that such clusters of traits normally composed one part of our human nature. The second originates from evolutionary pressures on the social aspects of human experience such as empathy, connectedness, altruism, mutuality cooperativeness and tribalism (i.e. collective aggressive territoriality and tribal exclusivity) fulfilling mainly the phenomenon of eusociality for our species [1]-[3]. These social aspects are deficient in various combinations or in individuals vulnerable for schizophrenia, as well as to a various degree, for other MMDs, especially for schizophrenia. Untreated symptoms include delusions, hallucinations both auditory and visual, disorganized speech, catatonic behavior, and negative symptoms, such as a lack of motivation, memory, personal care and occupational dysfunction [4]. Periods of prodromal or residual symptoms include, odd beliefs or disturbance of perception. Such deficits are often masked by learned civility. Preexisting, prominent traits for these individuals include self-absorption, dearth of empathy, aloofness, a tendency to be or act alone, a felt inner void or boredom, and an aversion to connect with others-all characteristics of the premorbid personality. Normally, as E.O. Wilson explains, we balance traits that are unamalgamated, and chimera-like. This makes us successful but conflicted social animals. He further states that we exist in unstable compromises between ourselves and those close to us within our own tribe and adjoining ones [1]-[3].

\subsection{The Significance of the Cluster of Gene Loci-Variants Associated with Schizophrenia}

Based on recent findings the cluster of gene loci-variants associated with schizophrenia [5] [6] does not directly lead to the disorder. The implicated genes may actually express themselves in lopsided, phenotypic traits comprising the premorbid personality. Such expression creates a pool of vulnerable individuals, some of whom will develop a MMD but at higher rate to those of the general population. Such occurrence is amenable to prediction only by statistical methods [7] [8]. The Genome-Wide Analysis was the largest so far to first claim that specific SNPs are significantly associated with a range of childhood-onset and adult-onset mental disorders. For the five major disorders investigated, SNPs at four loci on regions of chromosomes 3p21 and 10q24, and SNPs in two L-type, voltage-gated calcium-channel subunits, CACNA1C and CACNB2 exceeded the norm or genome-wide significance in their primary analysis [8].

\subsection{Extreme Temperamental Variance and Society}

Extreme lopsidedness in variability at the edge of the temperamental spectrum creates a group of mismatched individuals for the prevailing, social zeitgeist of the society in which they live. They are labeled as character disorders, or whatever the particular era they live in decides to call them [9]. These individuals, in addition to having personal dysphoria — often severe above and beyond that which are normally felt by all humans, such as a inner-void felt from within, boredom, dysthymia, irritability, aloofness, an aversion to social interaction and possibly others_-also have a dissonance between themselves and the prevailing but ever changing, circumstances, rules, and ethos of the social zeitgeist, and everyday ability to function. This often places them in conflict with the prevailing, social norm. These dysfunctional individuals while variably labeled "behaviorally troubled" or outright "character disorders", nevertheless, if also intelligent tenacious and curious, they can now express significant creativity [10]-[12]. Their particular temperamental variance acts as an enabling factor. For instance, an impulsive, periodically explosive, intense, fearless, ambitious, and intelligent man will be prized as a great warrior, like Alexander the Great [13]. Conversely, Steve Jobs, an intense, compulsive, tenacious visionary, created and shaped the future in information technology. In spite of it, he was kicked out for a time from his own company [14]. The gifted mathematician but socially odd, writer Lewis Carroll, author of Alice in Wonderland was merely deemed an eccentric scholar by the real Alice's family for his obsessive habit of photographing the eleven-year-old in the nude [15]! Today, L. Carroll would be labeled as a pedophile and recommended for treatment if not arrest. Michelangelo, Leonardo Da Vinci [16], Isaac Newton and from our times Nicola Tesla, R. 
Feynman [17], and Albert Einstein [18], all shared similar, temperamental peculiarities. The list is long. Conversely, individuals, rich in social attributes and traits, while contributing to the cohesiveness and harmony of the tribe, would not advance without the significant and fundamental, creative contribution from those lacking in these same traits [10].

\subsection{The Significance of Extreme Temperamental Variance in MMDs}

Individuals who are lopsided in temperament, creative or not, as mentioned, significantly also make up a pool of vulnerable individuals some of whom will develop major mental disorder at a much greater rate than the general population [5] [6]. Their aberrant traits are now renamed "premorbid personality" or "residual" following remission [19] [20]. The occurrence of a MMD for these individuals is probabilistic and amenable to prediction only by statistical methods. The rate for the general population is $3 \%-6 \%$ worldwide irrespective of culture for all three types combined [10]. The clinical expression of MMDs should be considered as a periodic, phenomenon superimposed on a patient's lopsided, genetic temperament. They are characterized by frequent remissions and relapses, at least upon onset, that later become permanent, akin to the occurrence of atrial fibrillation which becomes also permanent later on [21] [22]. For example, major depressive disorder is more common among first, generation relatives of people suffering from the same disorder than among the general population [23]. Results from family, adoption, and twin studies indicate that bipolar disorder is usually inherited. Nevertheless, the mode of transmission and its genetic relationship to other mood disorders has not been definitively identified or documented [24].

Significantly, symptoms of MMDs, in addition to the presence of a generic psychosis with delusions and hallucinations-to be addressed later-are made up of antithetical substitutes with an "either-or" character [25]-[27]. Importantly, compelling evidence accumulated from disparate fields indicates that in reality, there exists only one major mental disorder [8] [28]. It is expressed in three main clinical expressions with their own variability. These expressions frequently overlap or even alternate, resulting in a messy clinical picture, rarely, if ever conforming to the categorical guidelines of the now ICD-10 series. This is particularly evident in the case of ADHD, which later is often expressed as bipolar disorder. This phenomenon is traditionally termed co-morbidity, instead of being considered-as the clinical evidence suggests-another manifestation of the same MMD. These expressions include schizophrenia involving the coordination of thinking, feeling and behavior and appropriateness of social interactions such as biipolar affective disorder involving mood modulation, and OCD involving the algorithmic faculty (i.e., responsible for scheduling, sequencing, fore planning future actions, routines, and habits) [25] [29]. In addition, the anxiety disorders and phobias are also often a commonly shared symptom of all MMDs [29]. The symptoms of all of these syndromes are, as mentioned, characterized by antithetical substitutes, i.e., the manic-depressive oscillation characteristic of bipolar affective disorder, the ambivalence of schizophrenia (the rapid attitudinal oscillations towards a person or object), with its either-or, concretistic and non-sequitur thinking, inability to accommodate ambiguity, apathy, punctuated by explosive outrage and mismatched responses to a given social situation. In the case of OCD, over-diligence, switches to slovenliness along with the equivocation and inability to bring closure to a decision, replacing obsessive tenacity [25] [30] as well as entrainment of thoughts. Significantly, in the case of an intermittent, major, depression, the presence of a manic phase is overlooked occurring usually as an unnoticed, transient, emotional rush-readily recounted by the patient when asked. All point to disturbed coordination in the expression of the higher faculties.

\subsection{The Significance of the Overall Synchronizing Mode of Brain Function}

Actually, all of the aforementioned, clinical expressions manifest themselves as a kind of psychic-Parkinsonism [31] [32], reflecting an abrupt switch from the normal phase of the overall, coordinating mode of brain function, insuring smoothness, elegance and subtlety, to that of a black and white, either-or, and entrained character in their expressions. The normally present, over-all, synchronizing faculty of the expression of all higher mental functions is rarely taken into account. However, it is responsible for the elegant expression, characteristic of normal humans in action, true for all higher mental faculties, including the aforementioned areas of thinking, feeling, coordination of thinking with feelings, mood modulation and the algorithmic one responsible for scheduling, sequencing, and routine habit formation [32] [33]. During the onset of a MMD or during a relapse, this mode switches to a pathologically ordered state with familiar symptoms characterized by antithetical substitutes mentioned above. This phenomenon is particularly startling during clinical interactions in a flare up of the bor- 
derline disorder—a possible variant of bipolar disorder. The clinician witnesses within minutes rapid, antithetical expressions of feelings, attitudes and utterances by the sufferer [29] [34] [35]. It is akin to the cacophony ensued by a performing orchestra following the sudden departure of its conductor. Importantly, the co-occurrence of delusions and hallucinations can be interpreted separately, as an attempt by the integrating function of the brain to make sense of the underlying dysfunction, often similarly observed in many physical conditions such as fever, brain trauma, toxic states or following heart surgery to name a few-all successfully treated by the same anti-psychotic medications. The underlying, lopsided personality remains the same.

Conciliating findings point to a common, neural-developmental origin for all MMDs. Genetic loci associated with schizophrenia do not directly lead to the disorder. They code for the expression of lopsided temperamental variants in individuals, originating from mainly one part of our human nature. These individuals contribute to flexibility, and creative input to our species, concomitantly incur vulnerability for developing MMD as an evolutionary trade off. MMDs initially, are expressed as periodic, symptoms of an underlying temperamental, extreme variant that later becomes permanent. Underlying, aberrant traits remain unaltered. Clinical expressions are characterized by "either-or," antithetical substitutes, in addition to the co-occurring psychosis. The latter is common to other assaults on the brain function. These characteristic symptoms are the result of a disturbed, coordinating property of brain function, normally responsive for the smooth, synchronizing expression of all higher mental faculties.

\section{Conclusion}

This presented material suggests that studies of personality and temperamental types in their above described form are very important because based on the presented evidence, the same clusters of gene loci associated with a specific mental disorder such as schizophrenia ought to be found congregated in individuals manifesting the same clusters of traits that confer vulnerability to mental disorders at a much higher rate compared to the general population, but not invariably expressing the disorder [12]. The traditionally, recognized cluster of five personality traits including major depression (i.e., bipolar affective disorder), neurotic behavior, antagonism versus agreeableness (i.e., borderline personality disorder), extroversion versus introversion (i.e., schizophrenia), and conscientiousness versus openness to new experiences should all be renamed using a better term similar in meaning that better reflect their dual, evolutionary origin. Then, by modifying existing personality and temperament tests [27] [33], geneticists and clinicians alike will be better able to accurately identify vulnerable groups. This will enable researchers to discern patterns and relationships between genes, clusters of genes, and behavioral traits involved in the expression of a temperamental phenotype in individuals enabling creativity as well as a trade-off to susceptibility to a mental disorder. Importantly, this will also enable identification of circumstances and triggers that compromise the overall synchronous and coordinating faculty of brain functions for these populations that result for some in the periodic epiphenomenon which later usually become permanent as in the case of schizophrenia and other mental diseases. In addition to the clinical evidence cited, this synthesis is also based on the author's own longitudinal observations of over ten thousand patients, spanning five decades.

\section{References}

[1] Nowak, M.A., Wilson, E.O. and Tarnita, C.E. (2010) The Evolution of Eusociality. Nature, 466, 1057-1062. http://dx.doi.org/10.1038/nature09205

[2] Wilson, E.O. (1978) On Human Nature. Harvard University Press, Cambridge.

[3] Wilson, E.O. (2012) The Social Conquest of Earth. Pub. Liveright Corp., New York.

[4] Amann, B., Gomar, J.J., Ortiz-Gil, J., McKenna, P., Sans-Sansa, B., Sarró, S., et al. (2012) Executive Dysfunction and Memory Impairment in Schizoaffective Disorder: A Comparison with Bipolar Disorder, Schizophrenia and Healthy Controls. Psychological Medicine, 42, 2127-2135. http://dx.doi.org/10.1017/S0033291712000104

[5] Pediaditakis, N. (2015) Addressing a Conundrum: Why, According to the Genome-Wide Association Study and Psychiatric Genome Consortium Are Clusters of Gene Loci Variants Associated with Schizophrenia Found Scattered in Non-Schizophrenia Populations? A Consilience. International Journal of Molecular Genetics, 6, 21-29.

[6] Bergen, S.E. and Petryshen, T.L. (2012) Genome-Wide Association Studies (GWAS) of Schizophrenia: Does Bigger Lead to Better Results? Current Opinion in Psychiatry, 25, 76-82. http://dx.doi.org/10.1097/YCO.0b013e32835035dd

[7] Gershon, E.S., Alliey-Rodriguez, N. and Liu, C.Y. (2011) After GWAS: Searching for Genetic Risk for Schizophrenia and Bipolar Disorder. American Journal of Psychiatry, 168, 253-256. 
http://dx.doi.org/10.1176/appi.ajp.2010.10091340

[8] Cross-Disorder Group of the Psychiatric Genomic Consortiums (2013) Cross-Disorder Group of the Psychiatric Genomics Consortium. Identification of Risk Loci with Shared Effects on Five Major Psychiatric Disorders: A GenomeWide Analysis. The Lancet, 381, 1371-1379. http://dx.doi.org/10.1016/S0140-6736(12)62129-1

[9] Cooper, R. (2014) Diagnosing the Diagnostic and Statistical Manual of Mental Disorders Fifth Edition. Karnac Books, London.

[10] Pediaditakis, N. (2014) The Association Between Major Mental Disorders and Geniuses. The Psychiatric Times, 31, 1-4. http://www.psychiatrictimes.com/major-depressive-disorder/association-between-major-mental-disorders-and-geniuses

[11] Rothenberg, A. (1990) Creativity and Madness: New Findings and Old Stereotypes. Johns Hopkins University Press, Baltimore.

[12] Waddell, C. (1998) Creativity and Mental Illness: Is There a Link? Canadian Journal of Psychiatry, 43, 166-172.

[13] Fox, R.L. (2004) Alexander the Great. Penguin Books Ltd., London.

[14] Isaacson, W. (2013) Steve Jobs. Simon \& Schuster, New York.

[15] Woolf, J. (2010) The Mystery of Lewis Carroll: Discovering the Whimsical, Thoughtful, and Sometimes Lonely Man Who Created Alice in Wonderland. Mcmillan, New York.

[16] Davis, R.C. and Lindsmith, B. (2011) Renaissance People: Lives That Shaped the Modern Age. John Paul Getty Museum, Los Angeles.

[17] Krauss, L. (2011) The Quantum Man. W. W. Norton \& Company, Inc., New York.

[18] Issacson, W. (2007) Einstein: His Life and Universe. Gale Cengage Learning, New York.

[19] Solano, J.R. and González, D.C.M. (2000) Premorbid Personality Disorders in Schizophrenia. Schizophrenia Research, 44, 137-144. http://dx.doi.org/10.1016/S0920-9964(99)00203-0

[20] Bora, E., Gökçen, S., Kayahan, B. and Veznedaroglu, B. (2008) Deficits of Social-Cognitive and Social-Perceptual Aspects of Theory of Mind in Remitted Patients with Schizophrenia: Effect of Residual Symptoms. Journal of Nervous Mental Disorders, 196, 95-99. http://dx.doi.org/10.1097/NMD.0b013e318162a9e1

[21] Haro, J.M., Novick, D., Suarez, D., Alonso, J., Lépine, J.P., Ratcliffe, M. and the SOHO Study Group (2006) Remission and Relapse in the Outpatient Care of Schizophrenia: Three-Year Results from the Schizophrenia Outpatient Health Outcomes Study. Journal of Clinical Psychopharmacology, 26, 571-578. http://dx.doi.org/10.1097/01.jcp.0000246215.49271.b8

[22] Jahangir, A., Lee, V., Friedman, P.A., Trusty, J.M., Hodge, D.O., Kopecky, S.L., Packer, D.L., Hammill, S.C., Shen, W.-K. and Gersh, B.J. (2007) Long-Term Progression and Outcomes with Aging in Patients with Lone Atrial Fibrillation: A 30-Year Follow-Up Study. Circulation, 115, 3050-3056. http://dx.doi.org/10.1161/CIRCULATIONAHA.106.644484

[23] Warren, B.J. (2007) Mood Disorders: Management of Moods and Suicidal Behavior. Revised from Chapter 20 by Wood, S. and Bailey, K., p. 352. http://downloads.lww.com/wolterskluwer_vitalstream_com/sample-content/9780781791694_Boyd/Boydch20.pdf

[24] McCuffin, P., Rijsdijk, F., Andrew, J., Sham, P., Katz, R. and Cardno, A. (2003) The Heritability of Bipolar Affective Disorder and the Genetic Relationship to Unipolar Depression. Archives of General Psychiatry, 60, 497-502. http://dx.doi.org/10.1001/archpsyc.60.5.497

[25] Mayo Clinic Staff (2015) Obsessive-Compulsive Disorder (OCD). http://www.mayoclinic.org/diseases-conditions/ocd/basics/definition/con-20027827

[26] Pediaditakis, N. (2002) Borderline Phenomena Revisited: A Synthesis. The Psychiatric Times, 37-41.

[27] Laceulle, O.M., Ormel, J., Vollebergh, W.A., Van Aken, M.A. and Nederhof, E. (2014) A Test of the Vulnerability Model: Temperament and Temperament Change as Predictors of Future Mental Disorders-The TRAILS Study. Journal of Child Psychology and Psychiatry, 55, 227-236. http://dx.doi.org/10.1111/jcpp.12141

[28] Pediaditakis, N. (1998) Shared Characteristics in the Clinical Expression and Pharmacological Responses of Mental Disorders and Their Possible Collective Significance. Medical Hypotheses, 50, 347-352. http://dx.doi.org/10.1016/S0306-9877(98)90124-4

[29] Andreasen, N.C., Nopoulos, P., O’Leary, D.S., Miller, D.D., Wassink, T. and Flaum, M. (1999) Defining the Phenotype of Schizophrenia: Cognitive Dysmetria and Its Neural Mechanisms. Biological Psychiatry, 46, 908-920. http://dx.doi.org/10.1016/S0006-3223(99)00152-3

[30] Grassi, G., Pallanti, S., Cantisani, A. and Pellegrini, M. (2011) Obsessive-Compulsive Disorder Comorbidity: Clinical Assessment and Therapeutic Implications. Frontiers in Psychiatry, 2, 70. 
[31] Pediaditakis, N. (2012) Origins and Mechanisms in the Development of Major Mental Disorders: A Clinical Approach. Journal of Behavioral and Brain Science, 2, 269-275. http://dx.doi.org/10.4236/jbbs.2012.22030

[32] Uhlhaas, P.J. and Singer, W. (2006) Neural Synchrony in Brain Review Disorders: Relevance for Cognitive Dysfunctions and Pathophysiology. Neuron, 52, 155-168. http://dx.doi.org/10.1016/j.neuron.2006.09.020

[33] Jorgenson, E. (2015) Development of the Open Extended Jungian Type Scales 1.2. Creative Commons AttributionNonCommercial-ShareAlike 4.0 International License.

[34] Pediaditakis, N. (2006) Considering the Major Mental Disorders as Clinical Expressions of Periodic Pathological Oscillations of the Overall Operating Mode of Brain Function. Medical Hypotheses, 67, 395-400. http://dx.doi.org/10.1016/j.mehy.2005.09.015

[35] Pediaditakis, N. (1992) Deterministic Nonlinear Chaos in Brain Function and Borderline Psychopathological Phenomena. Medical Hypotheses, 39, 67-72. http://dx.doi.org/10.1016/0306-9877(92)90143-Z 